\title{
Genotyping of Giardia duodenalis isolates in asymptomatic children attending daycare centre: evidence of high risk for anthroponotic transmission
}

\author{
A. P. OliVEIRA-ARBEX, E. B. DAVID, T. C. G. OLIVEIRA-SEQUEIRA, \\ G. N. BITTENCOURT AND S. GUIMARÃES* \\ Parasitology Department, Institute of Bioscience, São Paulo State University (UNESP), Campus de Botucatu, \\ Botucatu, São Paulo, Brazil
}

Received 5 March 2015; Final revision 10 September 2015; Accepted 24 September 2015; first published online 23 November 2015

\section{SUMMARY}

Giardia duodenalis is a common intestinal parasite infecting children attending daycare centres. This study aimed to verify Giardia occurrence and the genotypes of isolates infecting children aged 0-6 years and workers at a daycare centre in the state of São Paulo, Brazil. The families of children who tested positive for Giardia, were asked to provide stool samples from household members and their dogs. Samples (123 children, 14 centre employees, 44 household members, 19 children after treatment, and $20 \mathrm{dogs}$ ) were examined for intestinal parasites using concentration methods. DNA extracted from all samples was submitted for polymerase chain reaction (PCR) testing and the amplicons generated were used for multilocus sequence typing of beta-giardin $(b g)$, triose phosphate isomerase (tpi) and glutamate dehydrogenase ( $g d h)$ genes. Giardia was detected in $15.9 \%$ and $28.6 \%$ of the 220 samples by microscopy and PCR, respectively. Analysis of sequences retrieved from 29 isolates revealed both assemblages A (31\%) and B (69\%). Subassemblages AII, BIII and BIV were identified and the alignment of the $b g, g d h$ and $t p i$ sequences revealed the presence of some single nucleotide polymorphisms, especially in assemblage B sequences. The higher predominance of assemblage B and the identification of the AII type support the view that anthroponotic transmission appears to be an important route of transmission in environments that concentrate children at an age when poor hygiene practices make them more vulnerable to such infection.

Key words: Anthroponotic, children, daycare, genotyping, Giardia duodenalis.

\section{INTRODUCTION}

Currently, as many children spend a lot of their time in a childcare facility, the shift to out-of-home care has had a significant impact on the attending child's health [1]. The attendance of younger children at

\footnotetext{
* Author for correspondence: Dr S. Guimarães, Parasitology Department, Institute of Bioscience, São Paulo State University (UNESP), Campus de Botucatu, Botucatu, São Paulo, PO Box 510; CEP: 18618-970, Brazil.

(Email: sgviana@ibb.unesp.br)
}

daycare centres increases the risk of acquiring infectious diseases when immune system immaturity and limited hygiene practice training make children more vulnerable to circulating infectious agents in the care environment $[2,3]$.

Faecal-oral transmitted infections occur frequently in daycare attendees and can be associated with diarrhoeal episodes [2, 3]. In such enclosed environments, the incidence of gastrointestinal disease episodes in children may be twice as high compared to homecared children $[4,5]$. Along with bacterial and viral 
enteropathogens, the protozoan Giardia duodenalis (syn. G. intestinalis, G. lamblia) stands out as one of the most common causes of diarrhoea in children attending daycare centres $[3,4,6]$. Giardiasis may remain asymptomatic or cause acute or chronic diarrhoea but currently, especially in developing areas, there is evidence that it may be associated with failure to thrive and nutrient deficiencies, leading to both growth and cognitive impairment during early childhood [7]. Regardless of the clinical impact, infected children are potential sources of cysts by which infection is transmitted to other children and adults in close contact [8]. According to Thompson, $20-25 \%$ of childcare staff and family members may become infected enabling the infection to reach the wider community [8].

Several community studies have revealed high prevalence rates of Giardia infection in children, especially in low-income populations. In South America, Giardia infection rates ranging from $11 \%$ to $30 \%$ have been previously documented in children of preschool age in some countries [9, 10], including Brazil $[11,12]$ where recent surveys have registered prevalence rates of nearly $50 \%[6,13]$ or even $100 \%$ [14].

Despite such high prevalence, a better understanding of the transmission dynamics of this parasite in different populations has been pursued over the years $[15,16]$. Recent advances in molecular techniques have allowed the recognition that $G$. duodenalis is a complex species comprising morphologically indistinguishable but genetically distinct isolates [17]. Human and animal isolates of $G$. duodenalis are characterized by at least eight distinct groups, referred to as assemblages or genotypes $\mathrm{A}-\mathrm{H}[15,16]$. Assemblages A and B are associated with human infections as well as a broad range of mammals, while the other assemblages have strong animal host specificities [15] although assemblages $\mathrm{C}-\mathrm{F}$ are occasionally isolated from human infections [15]. Therefore, although animals can harbour both zoonotic and host-specific assemblages, the zoonotic potential of Giardia still needs to be further assessed and clarified [16].

In recent years, molecular epidemiological studies have identified assemblages A and B associated with humans infections in different geographical locations and populations. In South America, most studies have focused on childhood infections and both assemblages have been identified $[9,10,18,19]$, with a predominance of assemblage B [9, 18, 19]. In Brazil, assemblages $\mathrm{A}$ and $\mathrm{B}$ have also been recovered from human infections in different populations including children [14, 20-23], hospital patients [22, 24, 25] and fishing village populations [26]. Assemblages A and $\mathrm{B}$ sequences have been further classified to subassemblages AI, AII, BIII and BIV. Genetic diversity has also been shown in isolates from companion animals [20, 22-26] as well as from environmental samples [22, 23]. Although companion animals usually harbour the host-adapted assemblages, i.e. C and $\mathrm{D}$ for dogs and $\mathrm{F}$ for cats, both assemblages $\mathrm{A}$ and $B[20,22-26]$ have been found in these hosts.

Given the need for a better understanding of the clinical epidemiology and public health significance of $G$. duodenalis infection, particularly in endemic areas, we here propose to estimate the prevalence of infection, and evaluate the genetic diversity of isolates, associated with daycare centre attendees and staff, as well as in household members and dogs living in close proximity with the children tested positive for Giardia cysts. By using a multilocus sequence typing approach, we aimed to assess the occurrence and frequency of the genotypes circulating in this particular group.

\section{MATERIAL AND METHODS}

\section{Study design, area and population}

The study was conducted from April 2012 to April 2013 in a daycare centre in Vitoriana, a district of Botucatu municipality, São Paulo State, Southeastern Brazil. Based on the last census in 2010 [27], Botucatu has 127328 inhabitants, about 3130 of whom live in Vitoriana which is located $\sim 16 \mathrm{~km}$ from the city; it is a low socioeconomic area although most of the population has access to safe drinking water and sanitation facilities. The population is attended by a primary healthcare facility and the daycare centre serves most pre-school age children from low-income families.

At the time of the study, 140 children aged 0-6 years were enrolled in the centre with 17 staff. Prior to the work, meetings were held with the daycare staff and the parents/guardians of children to inform them of the purpose and procedures of the study and request their collaboration. All attending children and daycare centre workers were invited to participate in the study and the parents were informed that when their child tested positive for Giardia, faecal samples would be requested from household members and their dogs. Further they were advised that all positive cases for Giardia and other pathogenic intestinal parasites should be treated with appropriate drugs at the local healthcare centre and 
following therapy completion, a new stool examination should be performed to ensure intestinal eradication. Informed consent was received for all study subjects prior to sample collection and parents/guardians were interviewed with a structured questionnaire covering demographic data (age, sex), socioeconomic status (parents'/guardians' occupation and education level, household income, number of adults and children in families) and, household and environmental conditions (hygiene habits, water supply, source of drinking water, sewage availability, latrine system, type of housing, and contact with household pets). The study was approved by the Research Ethics Committees of the Botucatu Medical School, UNESP and Animal Experimentation/Biosciences Institute/UNESP.

\section{Faecal samples and detection of $\boldsymbol{G}$. duodenalis cysts}

All children and staff in the daycare centre and their family members were provided with a package with three pots containing $2 \cdot 5 \%$ potassium dichromate solution, and instructed to collect the stool specimens on alternate days. At the laboratory, the samples from each subject were pooled, filtered through gauze into a centrifuge tube and washed three times with distilled water by centrifugation $(800 \mathrm{~g}$ for 3 $\mathrm{min})$ to remove potassium dichromate and to concentrate the material. A small amount of sediment was added to a slide with a drop of Lugol iodine and examined at $40 \times$ magnification under a light microscope. In addition, a portion of faecal sediment was processed by the zinc sulfate (specific gravity $1 \cdot 18$ ) flotation technique [28] and examined microscopically. A positive result from either of these methods was interpreted as indicative of Giardia infection. For dogs, a single faecal sample was collected in plastic vials, and processed as above.

\section{Treatment}

All study subjects positive for Giardia and/or other intestinal parasites positive cases were referred for treatment with a $400 \mathrm{mg}$ albendazol tablet/day, for 5 days. Children aged $<2$ years received $15-20 \mathrm{mg} / \mathrm{kg}$ metronidazole per day, for 5 days. At 21-30 days post-treatment, individuals were asked to provide three further stool samples, which were examined by the same procedures.

\section{DNA extraction}

DNA was extracted from all faecal samples regardless of the microscopic test results. To optimize disruption of the cysts, prior to DNA extraction, $200 \mathrm{mg}$ of centrifuged faecal sediment was subjected to three freezethaw cycles as follows: two cycles of $5 \mathrm{~min}$ in liquid nitrogen and then in a water bath at $70^{\circ} \mathrm{C}$, followed by one cycle in liquid nitrogen for $5 \mathrm{~min}$ and $95^{\circ} \mathrm{C}$ for 5 min. DNA was extracted from disrupted cysts using the QIAamp Stool mini kit (Qiagen, Germany) according to the manufacturer's instructions. All the DNA samples were stored at $-20^{\circ} \mathrm{C}$ until further analysis.

\section{Molecular and phylogenetic analysis}

All DNA samples were analysed by sequence-based genotyping using three gene loci beta-giardin $(b g)$, triose phosphate isomerase (tpi) and glutamate dehydrogenase $(g d h)$. Fragments were amplified with a nested $b g$ polymerase chain reaction (PCR) [29], a nested tpi PCR [30] and a semi-nested gdh PCR [31], which yielded the expected amplicons of 511, 530 and 432 $\mathrm{bp}$, respectively.

Positive PCR products were purified using spin columns (QIAquick PCR purification kit, Qiagen) and sequenced on both strands by Sequencing Service (Macrogen Inc., Seoul, Korea). Nucleotide sequences and chromatograms were analysed and edited using CLC Main Workbench software, version 7.0 (CLC Bio, Qiagen). Sequences from this study were aligned with each other and reference sequences downloaded from GenBank (listed below) using Clustal X [32]. The assemblages and sub-assemblages at each locus were identified by BLAST searches against the following reference sequences: $g d h$ gene (accession nos. L40509.1 AI, L40510.1 - AII，AF069059.1 - BIII，L40508.1 $\mathrm{BIV}$ ); $b g$ gene (accession nos. AY258617.1-AI, AY072723.1 - AII, AY072726.1 - BIII, AY072725.1 $\mathrm{BIV}$ ); tpi gene (accession nos. L02120.1-AI, U57897.1 - AII, AF069561.1 - BIII, L02116.1 - BIV). The nucleotide sequences obtained in the present study were submitted to GenBank and were assigned accession numbers KT334235-KT334293.

Phylogenetic analyses were performed with MEGA software v. 5.0 (www.megasoftware.net) and for each locus assessed, a phylogenetic tree was constructed using neighbour-joining and maximum likelihood algorithms. Bootstrap analysis was applied to evaluate the reliability of clusters by using 1000 replicates.

\section{Data analysis}

Statistical analyses were carried out using Epi Info software v. 7.0 (Centers for Disease Control and 
Prevention, USA). Data were summarized by absolute frequency and percentage. Differences between proportions were initially submitted to univariate analysis. $\chi^{2}$ test or Fisher's exact test were used for dichotomous variables and the Mann-Whitney test for numeric variables. Multivariate analysis (logistic regression) was performed using a change-in-estimate approach for selecting variables [33]. Briefly, a preliminary model included variables with $P<0 \cdot 1$ from the univariate analysis. Other models included also (one by one) all the other study variables. Variables were kept in the final model if they changed the odds ratio of any of the statistically significant values by $>10 \%$. $P<0.05$ was used to define statistical significance in all analyses.

\section{RESULTS}

\section{Characteristics of the study population and intestinal parasite status}

Of 140 children formally enrolled in the study, 123 provided the three faecal specimens, but of these children not all parents or guardians were present during the household-based interviews and hence the socioeconomic status could only be determined for $80 \cdot 5 \%$ (99/123). From the 123 daycare children aged 3-81 months participating in the study, $62(50 \cdot 4 \%)$ were male and $61(49 \cdot 6 \%)$ were female. The mean age was 48 (s.D. $\pm 1 \cdot 49$ ) months with 36- to 60-month-old children accounting for about $40 \%$ of the total. Based on 99 questionnaires, the socioeconomic data revealed that $89 \%$ of the children lived in brickwork houses and $100 \%$ had an indoor toilet. Concerning household sanitary conditions, $76 \cdot 8 \%$ of the houses had access to the public water supply and were connected to a public sewage system and, $91.9 \%$ had access to public garbage collection. In the houses outside the public water and sewerage services coverage area $(23 \cdot 2 \%)$, the families obtained water from artesian wells and disposed of sewage in septic tanks. Almost half $(43.4 \%)$ of the children lived in families with an income of about US\$200.00 per month. Approximately $70 \%$ of the parents or guardians had at least 6 years of formal education. Regarding family size, $67 \cdot 7 \%$ belonged to families with up three members. Almost all (91.4\%) households kept dogs as the main companion animal.

Microscopic examination revealed that at least one intestinal parasite was detected in $40 \cdot 6 \%(50 / 123)$ of the children attending daycare, with Giardia the most frequent at a prevalence rate of $21 \cdot 9 \%$ (27/123). Other intestinal parasites detected were: Entamoeba coli $(17 / 123,13 \cdot 8 \%)$; Blastocystis spp. (11/123, 8.9\%); and the helminths Enterobius vermicularis (2/123, 1.6\%), and one individual with Ascaris lumbricoides. According to information from parents/guardians and daycare centre staff, all children were considered asymptomatic, since no episodes of diarrhoea were reported in the 2 months prior to stool collection and during the study period.

Fourteen of 17 daycare workers provided faecal samples but all proved negative for intestinal parasites by microscopy. Only 16 household members of the 27 children who tested positive for Giardia agreed to provide faecal samples and thus specimens were obtained from 44 family members ( 31 adults, 13 children) and from 20 dogs. In these families, Giardia cysts were detected in samples from only two boys aged 17 and 90 months who were siblings of children attending the daycare centre. No dog was found positive for Giardia and the only intestinal parasite detected was the nematode Ancylostoma spp.

Thirty children in a daycare centre and two of their relatives were treated for Giardia and/or other pathogenic intestinal parasite infections. However, for follow-up post-treatment, only 19 of these submitted samples for examination. In seven samples, Giardia (six subjects) and Blastocystis (single subject) were detected after treatment, and none of the household members provided stool specimens for follow-up.

Analysis of prevalence of Giardia infection in daycare children according to age, gender and socioeconomic variables, showed no statistically significant differences. However, younger children had a higher risk of acquiring Giardia infection [odds ratio (OR) 0.69, 95\% confidence interval (CI) $00 \cdot 49-0 \cdot 97, P=0.03)$ as the risk of infection decreased with older age. In addition, children living in families with higher household densities were more likely to be infected (OR 1.8, 95\% CI 1.07-3.07, $P=0.03$ ). A higher risk was observed for 32 daycare children in households with $\geqslant 4$ members compared to those in households with fewer persons.

\section{Molecular detection and genotyping of Giardia isolates}

All Giardia microscopically positive and negative samples were submitted for PCR and a positive result was indicated when at least one of the three loci assessed was successfully amplified. As shown in Table 1, of the 35 Giardia microscopically positive samples (27 
Table 1. Detection of Giardia infection in daycare children, household members, care workers and dogs by microscopy and polymerase chain reaction (PCR)

\begin{tabular}{|c|c|c|c|}
\hline \multirow[b]{2}{*}{ Faecal samples } & \multirow[b]{2}{*}{ No. tested } & \multicolumn{2}{|c|}{ PCR results } \\
\hline & & $\begin{array}{l}\text { Positive } \\
(\%)^{*}\end{array}$ & $\begin{array}{l}\text { Negative } \\
(\%)\end{array}$ \\
\hline Children & 123 & & \\
\hline Positive $(n=27)$ & & $22(81 \cdot 5)$ & $05(18 \cdot 5)$ \\
\hline Negative $(n=96)$ & & $20(20 \cdot 9)$ & $76(79 \cdot 1)$ \\
\hline Household members & 44 & & \\
\hline Positive $(n=02)$ & & $02(100 \cdot 0)$ & 0 \\
\hline Negative $(n=42)$ & & $12(28 \cdot 6)$ & $30(71 \cdot 4)$ \\
\hline Care workers & 14 & & \\
\hline Positive $(n=0)$ & & 0 & 0 \\
\hline Negative $(n=14)$ & & 0 & $14(100 \cdot 0)$ \\
\hline Follow-up & 19 & & \\
\hline Positive $(n=06)$ & & $05(83 \cdot 3)$ & $01(16 \cdot 7)$ \\
\hline Negative $(n=13)$ & & $02(15 \cdot 4)$ & $11(84 \cdot 6)$ \\
\hline Dogs & 20 & & \\
\hline Positive $(n=0)$ & & 0 & 0 \\
\hline Negative $(n=20)$ & & 0 & $20(100 \cdot 0)$ \\
\hline Total & 220 & & \\
\hline Positive $(n=35)$ & & $29(82 \cdot 9)$ & $06(17 \cdot 1)$ \\
\hline Negative $(n=185)$ & & $34(18 \cdot 4)$ & $151(81 \cdot 6)$ \\
\hline
\end{tabular}

* Amplification at least one of the three loci assessed.

from children attending daycare, two from household members and six from follow-up), 29 were successfully amplified. Of the 185 samples previously identified as negative for microscopy, PCR products were generated in 34 of them (20 from daycare children, 12 from household members and two from follow-up). No amplicons were obtained from the daycare workers' and dogs' samples. Thus, in total, the presence of Giardia was detected in $63(28.6 \%)$ and 35 $(15.9 \%)$ of the 220 samples examined by PCR and microscopy, respectively.

Of the 63 PCR-positive samples, 37 showed products for a single gene, 12 for two genes and 14 for all three genes analysed. For each locus, amplicons were generated in $45(71 \%), 35(56 \%)$ and $23(37 \%)$ of the samples for $t p i, g d h$ and $b g$, respectively. Sequence analysis on 59 PCR products generated from 29 isolate/subject samples identified 15 sequences for $b g, 25$ for $t p i$ and 19 for $g d h$ and, of these isolates, 11 were successfully genotyped at all three loci, eight at two loci and 10 at a single locus (Table 2). Furthermore, sequences of these isolates were unambiguously identified as assemblages $\mathrm{A}$ or $\mathrm{B}$ by at least one of the three loci (Table 2, Supplementary
Table 2. Genotypic characterization of Giardia duodenalis isolates from children attending a daycare centre and household members

\begin{tabular}{|c|c|c|c|c|c|}
\hline \multirow[b]{2}{*}{ Isolate } & \multirow{2}{*}{$\begin{array}{l}\text { Age } \\
\text { (months) }\end{array}$} & \multirow[b]{2}{*}{ Sex } & \multicolumn{3}{|c|}{ Sub-assemblages } \\
\hline & & & $b g$ & tpi & $g d h$ \\
\hline DC01 & 76 & $\mathrm{~F}$ & BIV & BIV & BIV \\
\hline DC16 & 14 & M & AII & AII & AII \\
\hline DC18 & 66 & $\mathrm{~F}$ & AII & AII & - \\
\hline DC28 & 17 & M & - & BIV & - \\
\hline DC29 & 15 & $\mathrm{~F}$ & - & - & BIV \\
\hline DC33 & 56 & $\mathrm{~F}$ & AII & - & - \\
\hline DC34 & 28 & $\mathrm{~F}$ & BIV & BIV & - \\
\hline DC35 & 48 & $\mathrm{~F}$ & AII & AII & AII \\
\hline DC38 & 39 & $\mathrm{~F}$ & BIV & - & BIV \\
\hline DC39 & 36 & M & BIII & BIII & BIV \\
\hline DC41 & 52 & M & - & BIV & - \\
\hline DC42 & 25 & $\mathrm{~F}$ & - & BIV & - \\
\hline DC43 & 18 & M & - & BIV & BIV \\
\hline DC43 F-up & - & M & BIV & BIV & BIV \\
\hline DC46 & 28 & $\mathrm{~F}$ & - & BIV & \\
\hline DC50 & 52 & M & BIII & BIII & BIV \\
\hline DC56 & 25 & $\mathrm{~F}$ & - & AII & AII \\
\hline DC59 & 39 & $\mathrm{~F}$ & - & BIII & BIV \\
\hline DC90 & 24 & M & - & BIV & - \\
\hline DC110 & 64 & M & AII & AII & AII \\
\hline DC110 F-up & - & M & - & BIV & BIV \\
\hline HM110A & 17 & M & BIV & BIV & BIV \\
\hline HM110B & Adult & $\mathrm{F}$ & - & BIV & BIV \\
\hline DC111 & 13 & M & AII & AII & AII \\
\hline DC112 & 25 & M & - & BIV & - \\
\hline DC112 F-up & - & M & - & - & BIV \\
\hline HM112A & 90 & M & - & BIV & - \\
\hline DC113 & 32 & $\mathrm{~F}$ & AII & AII & AII \\
\hline DC113 F-up & - & $\mathrm{F}$ & AII & AII & AII \\
\hline
\end{tabular}

DC, Daycare isolate; HM, household member isolate; F-up, follow-up (new stool sample provided after treatment).

Figs S1-S3). Twenty (69\%) isolates were classified as assemblage B (14 from children, three from household members and three from follow-up test) and the assignment at each locus was seven at the $b g, 17$ at $t p i$ and 12 at $g d h$ (Table 2). The remaining nine isolates $(31 \%)$ recovered from eight daycare children (eight samples at baseline and one post-treatment) were typed as assemblage A (8 bg, 8 tpi, 7 gdh). Phylogenetic analysis confirmed the distribution of isolates into distinct clusters (Supplementary Figs S4-S6). The comparison of sequences obtained for $b g$, $t p i$ and $g d h$ and assigned as assemblages $\mathrm{A}$ and $\mathrm{B}$ revealed that $85 \%$ of them (50/59) showed $100 \%$ identity with reference sequences previously described; the remaining $15 \%(9 / 59)$ showed identity of $98 \%$ or $99 \%$. 
All assemblage A isolates were identified as subassemblage AII, and of assemblage B isolates, 17 were identified as BIV and three could not be clearly subtyped (Table 2). Thus, at $b g$ and tpi loci, these three isolates (DC39, DC50, DC59) were identified as BIII and at the $g d h$ locus they corresponded to BIV, exhibiting an inconsistency in the separation of these subgroups.

Single-nucleotide polymorphism (SNP) analysis at all loci revealed in some assemblage $\mathrm{B}$ sequences at least one nucleotide substitution compared to reference sequences (Table 3, Supplementary Figs S1S3). Further, some nucleotide substitutions at specific positions were found to discriminate between BIII and BIV sub-assemblages at all loci in the three isolates not clearly subtyped as BIII or BIV (Table 3). No overlapping nucleotide sequences and double chromatogram peaks at specific positions were observed. Of the assemblage AII isolates, only two sequences at the tpi locus showed a nucleotide substitution at position 879 (Supplementary Fig. S3).

In the context of the daycare centre, a particular situation was evident in relation to isolates from the same child that were recovered from samples collected at baseline (DC110) and at follow-up (DC110 F-up $_{1}$ post-treatment; in the former assemblage A (AII) was identified and in the latter, assemblage B (BIV) (Table 2). Interestingly, the isolates recovered from the samples from two household members (HM110A, HM110B) of this child corresponded to sub-assemblage BIV (Table 2); these sequences (DC110 F-up, HM110A, HM110B) were identical on alignment.

The distribution of the typed isolates from children, according to the daycare classrooms is reported in Table 4 . With the exception of classroom 1 which comprised the younger children, assemblage B was the most frequent in all other groups. Of the few household members that provided stool samples, isolates were recovered from three samples: two from individuals (one adult, one child) that lived with child DC110 in classroom 6 (mean age 60 months and only sub-assemblage AII identified) and, the other sample from a child relative of DC112 in classroom 4 (mean age 32.8 months and both subassemblages AII and BIV identified).

\section{DISCUSSION}

Enteroparasitic infections persist as neglected diseases that can lead to adverse consequences, particularly for children of preschool age living in low socioeconomic areas. In this study, intestinal parasitic infections were investigated in children attending a daycare centre and Giardia was the most frequent parasite detected $(21.9 \%)$, corroborating the findings reported in other investigations [14, 34-36]. Furthermore, in this population, it was found that younger children and children living within a family with $\geqslant 4$ members were at an increased risk for Giardia infection. The association between children's age and family size with Giardia infection has been previously reported [35, 37], and this reinforces the possibility of interpersonal transmission in a more susceptible and exposed group.

Recent advances in molecular techniques have provided important tools not only for detecting Giardia infection but also for understanding the epidemiology of transmission in target groups. We therefore investigated the genetic diversity of $G$. duodenalis infection in daycare attendees using three widely used markers, the $b g$, tpi and $g d h$ genes. Giardia positive and negative samples by microscopy were submitted to PCR reactions and amplification was achieved in $28.6 \%$ of samples compared to $15.9 \%$ by microscopy. It follows that reliance of detection by microscopy alone may significantly underestimate true parasite prevalence. Nevertheless, it is noteworthy that 6/35 microscopy-positive samples found here failed to show amplification products for $b g$, $g d h$ and tpi genes despite the proven higher sensitivity and specificity of such methods over conventional techniques; similar results have been reported in other Giardia molecular-based studies [38, 39]. These failures to isolate DNA or false-negative amplification from stools may be due to the presence of PCR inhibitors, differences in composition of samples and storage conditions [40]. The exclusion of these factors can be difficult but alternatives such as the use of extraction kits and the choice of an appropriate fixative suspension media (potassium dichromate or ethanol) may help minimize them.

Regarding PCR-positive reactions, different rates of amplification for the three loci were found with amplicons generated in $45(71 \%), 35(56 \%)$ and $23(37 \%)$ of the samples for $t p i, g d h$ and $b g$, respectively. These genes have been frequently used by others to detect and/or genotype Giardia isolates in stool samples, but differences in their performance have been reported [38, 39]. The reasons for this are unclear, but according to Broglia et al. [39] as these markers are all single-copy genes the different rates are not explained by a difference in target copy number. However, these authors emphasize that the presence 
Table 3. Polymorphic sites in the $\mathrm{gdh}, \mathrm{bg}$ and tpi sequences in Giardia duodenalis assemblage B isolates

\begin{tabular}{|c|c|c|c|c|c|c|c|c|c|c|c|c|c|}
\hline \multirow{2}{*}{$\begin{array}{l}\text { Assemblage } \\
\text { Glutamate de }\end{array}$} & \multirow{2}{*}{$\begin{array}{l}\text { Isolate/Genbank number } \\
\text { hydrogenase }(g d h)\end{array}$} & \multicolumn{12}{|c|}{ Nucleotide position } \\
\hline & & \multirow{2}{*}{$\begin{array}{l}109 \\
C\end{array}$} & \multirow{2}{*}{$\frac{154}{G}$} & \multirow{2}{*}{$\frac{157}{\mathrm{~T}}$} & \multirow{2}{*}{$\begin{array}{l}229 \\
\mathbf{T}\end{array}$} & \multirow{2}{*}{$\begin{array}{l}247 \\
\mathbf{T}\end{array}$} & \multirow{2}{*}{$\begin{array}{l}308 \\
G\end{array}$} & \multirow{2}{*}{$\begin{array}{l}340 \\
\mathbf{T}\end{array}$} & \multirow{2}{*}{$\begin{array}{l}361 \\
\mathrm{C}\end{array}$} & \multirow{2}{*}{$\begin{array}{l}370 \\
C\end{array}$} & \multirow{2}{*}{$\frac{397}{\mathrm{C}}$} & \multirow{2}{*}{$\begin{array}{l}406 \\
C\end{array}$} & \multirow{2}{*}{$\frac{412}{\mathbf{G}}$} \\
\hline BIII & AF069059.1 & & & & & & & & & & & & \\
\hline \multirow[t]{7}{*}{ BIV } & L40508.1 & $\mathbf{T}$ & . & . & C & C & . & $\mathrm{C}$ & $\mathbf{T}$ & . & . & . & A \\
\hline & DC39/KT334248 & $\mathrm{T}$ & $A$ & . & $\mathrm{C}$ & $\mathrm{C}$ & . & $\mathrm{T}$ & $\mathrm{T}$ & . & . & . & A \\
\hline & DC50/KT334251 & $\mathrm{T}$ & $A$ & . & $\mathrm{C}$ & $\mathrm{C}$ & . & $\mathrm{T}$ & $\mathrm{T}$ & . & . & & A \\
\hline & DC59/KT334252 & $\mathrm{T}$ & . & $\mathrm{C}$ & $\mathrm{C}$ & $\mathrm{C}$ & $A$ & $\mathrm{~T}$ & $\mathrm{C}$ & . & . & $T$ & A \\
\hline & HM110A/KT334243 & $\mathrm{T}$ & . & $\mathrm{C}$ & $\mathrm{C}$ & $\mathrm{C}$ & . & $\mathrm{T}$ & $\mathrm{C}$ & $T$ & $T$ & & A \\
\hline & DC110F-up/KT334242 & $\mathrm{T}$ & . & C & $\mathrm{C}$ & $\mathrm{C}$ & . & $\mathrm{T}$ & $\mathrm{T}$ & & & & A \\
\hline & HM110B/KT334244 & $\mathrm{T}$ & . & C & $\mathrm{C}$ & $\mathrm{C}$ & . & $\mathrm{T}$ & $\mathrm{C}$ & $T$ & $T$ & & A \\
\hline \multicolumn{2}{|c|}{ Beta-giardin $(b g)$} & 161 & 167 & 233 & 284 & 287 & 314 & 317 & 329 & 398 & 476 & & \\
\hline \multirow{8}{*}{$\begin{array}{l}\text { BIII } \\
\text { BIV }\end{array}$} & AY072726.1 & $\mathrm{G}$ & C & G & $\mathrm{C}$ & C & C & C & $\mathrm{C}$ & C & $\mathrm{T}$ & & \\
\hline & AY072725.1 & & $\mathbf{T}$ & A & . & $\mathbf{T}$ & $\mathbf{T}$ & $\mathbf{T}$ & & $\mathbf{T}$ & & & \\
\hline & DC01/KT334287 & $A$ & $\mathrm{~T}$ & A & . & $\mathrm{C}$ & $\mathrm{T}$ & $\mathrm{T}$ & $T$ & $\mathrm{~T}$ & $C$ & & \\
\hline & DC34/KT334288 & $\boldsymbol{A}$ & $\mathrm{T}$ & A & . & $\mathrm{C}$ & $\mathrm{T}$ & $\mathrm{T}$ & $T$ & $\mathrm{~T}$ & $C$ & & \\
\hline & DC38/KT334289 & $A$ & $\mathrm{~T}$ & A & . & $\mathrm{C}$ & $\mathrm{T}$ & $\mathrm{T}$ & $T$ & $\mathrm{~T}$ & $C$ & & \\
\hline & DC39/KT334293 & . & $\mathrm{C}$ & A & $T$ & $\mathrm{C}$ & $\mathrm{C}$ & $\mathrm{T}$ & $T$ & $\mathrm{C}$ & & & \\
\hline & DC43 F-up/KT334290 & $A$ & $\mathrm{~T}$ & A & . & $\mathrm{C}$ & $\mathrm{T}$ & $\mathrm{T}$ & $T$ & $\mathrm{~T}$ & $C$ & & \\
\hline & DC50/KT334291 & . & $\mathrm{C}$ & G & . & $\mathrm{C}$ & $\mathrm{T}$ & $\mathrm{T}$ & . & $\mathrm{C}$ & . & & \\
\hline \multicolumn{2}{|c|}{ Triose phosphate isomerase (tpi) } & 83 & 160 & 163 & 187 & 205 & 289 & 424 & & & & & \\
\hline BIII & AF069561.1 & C & C & C & A & G & A & G & & & & & \\
\hline \multirow[t]{18}{*}{ BIV } & L02116.1 & $\mathbf{T}$ & $\mathbf{T}$ & $\mathbf{T}$ & . & A & . & . & & & & & \\
\hline & DC01/KT334262 & $\mathrm{T}$ & $\mathrm{T}$ & $\mathrm{T}$ & . & A & . & $A$ & & & & & \\
\hline & DC28/KT334263 & $\mathrm{T}$ & $\mathrm{T}$ & $\mathrm{T}$ & . & A & . & $A$ & & & & & \\
\hline & DC34/KT334264 & $\mathrm{T}$ & $\mathrm{T}$ & $\mathrm{T}$ & . & A & . & $\boldsymbol{A}$ & & & & & \\
\hline & DC39/KT334265 & $\mathrm{T}$ & $\mathrm{C}$ & $\mathrm{C}$ & $\boldsymbol{G}$ & A & . & . & & & & & \\
\hline & DC41/KT334266 & $\mathrm{T}$ & $\mathrm{T}$ & $\mathrm{T}$ & . & A & . & $A$ & & & & & \\
\hline & DC42/KT334267 & $\mathrm{T}$ & $\mathrm{T}$ & $\mathrm{T}$ & . & A & . & $A$ & & & & & \\
\hline & DC43/KT334268 & $\mathrm{T}$ & $\mathrm{T}$ & $\mathrm{T}$ & . & A & . & $A$ & & & & & \\
\hline & DC43F-up/KT334269 & $\mathrm{T}$ & $\mathrm{T}$ & $\mathrm{T}$ & . & A & . & $A$ & & & & & \\
\hline & DC46/KT334270 & $\mathrm{T}$ & $\mathrm{T}$ & $\mathrm{T}$ & . & A & & $A$ & & & & & \\
\hline & DC50/KT334271 & $\mathrm{C}$ & $\mathrm{C}$ & $\mathrm{C}$ & . & $\mathrm{G}$ & $\boldsymbol{G}$ & . & & & & & \\
\hline & DC59/KT334272 & $\mathrm{C}$ & $\mathrm{T}$ & $\mathrm{T}$ & . & $\mathrm{G}$ & . & . & & & & & \\
\hline & DC90/KT334273 & $\mathrm{T}$ & $\mathrm{T}$ & $\mathrm{T}$ & . & A & . & $A$ & & & & & \\
\hline & HM110 F-up/KT334274 & $\mathrm{T}$ & $\mathrm{T}$ & $\mathrm{T}$ & . & A & . & $A$ & & & & & \\
\hline & HM110A/KT334275 & $\mathrm{T}$ & $\mathrm{T}$ & $\mathrm{T}$ & . & A & . & $A$ & & & & & \\
\hline & HM110B/KT334276 & $\mathrm{T}$ & $\mathrm{T}$ & $\mathrm{T}$ & . & A & . & $A$ & & & & & \\
\hline & DC112/KT334277 & $\mathrm{T}$ & $\mathrm{T}$ & $\mathrm{T}$ & . & A & . & $A$ & & & & & \\
\hline & HM112A/KT334278 & $\mathrm{T}$ & $\mathrm{T}$ & $\mathrm{T}$ & . & A & . & $A$ & & & & & \\
\hline
\end{tabular}

Accession numbers in bold are references sequences from GenBank. Dots indicate identity to the reference sequences. Novel substitution positions are highlighted in bold italics.

of mismatches between the genomic sequences and the primers used for PCR, may result in a marked reduction, or even a lack of amplification.

Our results showed that the successfully sequenced isolates were unambiguously assigned to assemblages A or B, with predominance of the latter (20/29). In recent years, molecular epidemiological studies have reported assemblages A and B associated with human infections in different geographical locations and populations. Similar to our study, the predominance of assemblage B, including in children, has been reported by other Brazilian authors [14, 23] as well as in investigations performed in different countries of Latin America such as Nicaragua [41], Argentina [9], Ecuador [18], Colombia [19]. By contrast, Souza et al. [24] and Kohli et al. [21] in Brazil found that infections with assemblage A were more prevalent, while Volotão et al. [20], also in Brazil, 
Table 4. Distribution of Giardia duodenalis assemblages according to daycare

\begin{tabular}{|c|c|c|c|c|}
\hline \multirow[b]{2}{*}{ Classroom isolate } & \multirow{2}{*}{$\begin{array}{l}\text { Age, mean } \\
\text { (months) }\end{array}$} & \multicolumn{3}{|c|}{ Sub-assemblage } \\
\hline & & $b g$ & $t p i$ & $g d h$ \\
\hline \multicolumn{5}{|l|}{ Class 1} \\
\hline DC16 & 14 & AII & AII & AII \\
\hline \multicolumn{5}{|l|}{ Class 2} \\
\hline DC28 & & - & BIV & - \\
\hline DC29 & $19 \cdot 5$ & - & - & BIV \\
\hline DC43 & & - & BIV & BIV \\
\hline DC46 & & - & BIV & \\
\hline \multicolumn{5}{|l|}{ Class 3} \\
\hline DC34 & & BIV & BIV & - \\
\hline DC42 & & - & BIV & - \\
\hline DC56 & $25 \cdot 5$ & - & AII & AII \\
\hline DC59 & & - & BIII & BIV \\
\hline DC90 & & - & BIV & - \\
\hline DC111 & & AII & AII & AII \\
\hline \multicolumn{5}{|l|}{ Class 4} \\
\hline DC38 & & BIV & - & BIV \\
\hline DC39 & $32 \cdot 8$ & BIII & BIII & BIV \\
\hline DC112 & & - & BIV & - \\
\hline DC113 & & AII & AII & AII \\
\hline \multicolumn{5}{|l|}{ Class 5} \\
\hline DC50 & & BIII & BIII & BIV \\
\hline DC41 & $50 \cdot 5$ & - & BIV & - \\
\hline DC35 & & AII & AII & AII \\
\hline \multicolumn{5}{|l|}{ Class 6} \\
\hline DC33 & 60 & AII & - & - \\
\hline DC110 & & AII & AII & AII \\
\hline \multicolumn{5}{|l|}{ Class 7} \\
\hline DC01 & & BIV & BIV & BIV \\
\hline DC18 & 71 & AII & AII & - \\
\hline
\end{tabular}

DC, Daycare isolate.

observed that this genotype was the only one infecting humans. On the other hand, similar prevalence of assemblages $\mathrm{A}$ and $\mathrm{B}$ have been detected in children in Cuba [42] and in inhabitants of fishing villages in Brazil [26]. Despite the weight of evidence, various factors need to be carefully considered to establish the prevalence and geographical distribution of these genetic groups, as methodological aspects (targeted genes, number of loci, primers, downstream procedures, etc.) may influence the findings $[15,16]$.

In this study, in the 29 isolates assessed, subassemblage determination was clearly achieved for 26 of them, including nine AII and 17 BIV. Interestingly, in recent studies in Brazil [22, 23] and Colombia [19], subtype BIV has been reported to be the most frequent sub-assemblage related to human infections, including in children [19], and environmental contamination of sources such as water and vegetables [22, 23]. The higher frequency of assemblage B isolates associated with infections in daycare children is an interesting finding and has been reported in other investigations [14, 22]. Although this genetic group has been identified in isolates from some animals, it is more frequently reported in human infections, and therefore it is closely related to anthroponotic transmission. In the context of a daycare centre, such transmission may play a role in the dissemination of Giardia cysts in children attending a particular environment where specific epidemiological characteristics may increase the risks for the parasite's transmission. In addition to the predominance of assemblage $\mathrm{B}$, all the assemblage A isolates were classified as AII, a subgroup that has been commonly associated with human infections. In fact, the two most common subassemblages, AI and AII, differ in host preference; however, humans have been frequently found infected with AII isolates [15].

Considering that in the course of the investigation, all children were characterized as asymptomatic, attempts to verify a likely association between assemblages and symptoms were not considered. To the best of our knowledge, findings on possible associations between assemblages and clinical status are still inconsistent [15]. Even so, interestingly some previous investigations had reported a correlation between assemblage $\mathrm{A}$ and symptomatic infections and between assemblage $\mathrm{B}$ and asymptomatic giardiasis [43-47]. In the context of our results, it is pertinent to emphasize that asymptomatic individuals play a role as carriers, spreading cysts both inside daycare and in their households, increasing the chances to infect other children and adults. This situation, when associated with poor hygiene practices may be crucial for Giardia transmission. Recently, a study performed with children living in a low socioeconomic community in northeastern Brazil [21], observed that children infected with isolates classified as assemblage B demonstrated a higher level of cyst shedding and these hosts could easily promote parasite spreading.

An interesting finding here was that three assemblage B isolates were not clearly subtyped at $b g$ and tpi loci but they were identified as BIII and at $g d h$ as BIV. SNP analysis at all loci revealed nucleotide substitutions at specific positions that discriminate between sub-assemblages BIII and BIV. These findings could be suggestive of mixed subtype infections or, to a lesser extent, genetic recombination, but further investigation would require cloning of PCR products. The inconsistency in typing results seems to be more 
related to mixed infections, mainly in infected individuals living in low-income areas [16]. Albeit in high prevalence, the occurrence of mixed infections is not easy to detect by conventional PCR assays, as the most abundant parasite population is preferentially amplified [48]. Recently a new RT-PCR assay based on assemblage-specific primers has been proposed [48] which might offer a more sensitive and precise tool for the detection of mixed infections. In view of our findings, it is probable that the high degree of polymorphism in assemblage B may make it difficult for the consistent determination of sub-assemblages within this group [49]. For this reason, it is possible that the genes currently used for genotyping, such as $t p i, g d h$ and $b g$ will be unable to consistently define the subassemblages because this group is too genetically diverse and the high substitution rate of these genes may obscure the true sub-assemblage patterns [49].

In the present study, the low participation of the families of Giardia-infected children was a limitation, hampering the detection of isolates associated with the household members' infections and the possibility of household transmission. Nevertheless, the observation that two genetically different isolates were recovered from the same child before (AII) and after (BIV) treatment is of interest and supports the plausibility of household transmission as the same types were identified in household members. Furthermore, the daycare child attended a classroom where other positive children were infected with AII isolates. Moreover, the scenario of possible reinfection of this child after treatment cannot be discounted.

Here, the predominance of assemblage B suggests that humans are the major source of Giardia cysts and that human-to-human transmission is a relevant route. In low-income areas, the lack of sanitation facilities favours the spread of cysts in the environment and potential contamination of water sources. The current study was conducted in such a community but where most of the children have access to safe drinking water and sanitation facilities. However, a lack of hand washing habit undoubtedly plays a major role in the spread and transmission of Giardia cysts through food handling by infected persons or directly by person-to-person contact.

In conclusion, despite the low participation of families and relatively small number of isolates examined the results presented herein outline pertinent aspects about the occurrence of $G$. duodenalis assemblages in children attending daycare centres and also provide some insights for future elucidation of giardiasis epidemiology in endemic areas. The study highlights the importance of investigating $G$. duodenalis genetic diversity for a more precise understanding of its epidemiology, while also focusing on improving children's health through prevention and control of giardiasis.

\section{SUPPLEMENTARY MATERIAL}

For supplementary material accompanying this paper visit http://dx.doi.org/10.1017/S0950268815002514.

\section{ACKNOWLEDGEMENTS}

This work was supported by the Fundação de Amparo à Pesquisa do Estado de São Paulo (FAPESP) through a research grant to S.G. (2011/ 52100-3). Graduate fellowships were granted to E.B.D. and A.P.O.A. by FAPESP (DD 2011/09963-0) and Coordenação de Aperfeiçoamento de Pessoal de Nível Superior (CAPES, Tropical Diseases Program), respectively. The authors thank all the families that participated in the study and the daycare staff. We also thank Ms. Patrícia Borim for technical support, Dr Carlos Magno Fortaleza and Dr José Eduardo Corrente for statistical assistance and Dr José Ricardo Jensen for language advice.

\section{DECLARATION OF INTEREST}

None.

\section{REFERENCES}

1. Enserink R, et al. Gastrointestinal and respiratory illness in children that do and do not attend child day care centers: a cost-of-illness study. PLoS ONE 2014; 8: 104-940.

2. Mink CM, et al. Infections in child-care facilities and schools. Pediatrics in Review 2009; 30: 259-269.

3. Enserink R, et al. Risk factors for gastroenteritis in child day care. Epidemiology and Infection 2015; 16: 1-14.

4. Thompson RCA. Giardiasis as a re-emerging infectious disease and its zoonotic potential. International Journal for Parasitology 2000; 12: 1259-1267.

5. Enserink R, et al. Infectious disease burden related to child day care in the Netherlands. Pediatric Infectious Disease Journal 2013; 32: 334-340.

6. Castro ED, et al. Enteropathogens detected in a daycare center, southeastern Brazil: Bacteria, Virus, and Parasite Research. Revista do Instituto de Medicina Tropical de Sao Paulo 2015; 57: 27-32. 
7. Halliez MC, Buret A. Extra-intestinal and long term consequences of Giardia duodenalis infections. World Journal of Gastroenterology 2013; 19: 8974-8985.

8. Thompson SC. Giardia lamblia in children and the child care setting: a review of the literature. Journal of Paediatrics and Child Health 1994; 30: 202-209.

9. Molina N, et al. High prevalences of infection with Giardia intestinalis genotype B among children in urban and rural areas of Argentina. Annals of Tropical Medicine and Parasitology 2011; 105: 299-309.

10. Rodríguez V, et al. Giardia duodenalis genotypes found in the Instituto Colombiano de Bienestar Familiar day care centres and dogs in Ibagué, Colombia. Biomédica 2014; 34: 271-281.

11. Silva RR, et al. Association between nutritional status, environmental and socio-economic factors and Giardia lamblia infections among children aged 6-71 months in Brazil. Transactions of the Royal Society of Tropical Medicine and Hygiene 2007; 5: 512-519.

12. Teixeira JC, Heller L, Barreto ML. Giardia duodenalis infection: risk factors for children living in sub-standard settlements in Brazil. Cadernos de Saúde Pública 2007; 23: $1489-1493$.

13. Santos CK, et al. Epidemiological, parasitological and molecular aspects of Giardia duodenalis infection in children attending public day care centers in southeastern Brazil. Transactions of the Royal Society of Tropical Medicine and Hygiene 2012; 8: 473-479.

14. Lima-Junior OA, et al. High occurrence of giardiasis in children living on a 'landless farm workers' settlement in Araras, São Paulo, Brazil. Revista do Instituto de Medicina Tropical de Sao Paulo 2013; 55: 185-188.

15. Feng Y, Xiao L. Zoonotic potential and molecular epidemiology of Giardia species and giardiasis. Clinical Microbiology Reviews 2011; 24: 110-140.

16. Ryan U, Cacciò SM. Zoonotic potential of Giardia. International Journal for Parasitology 2013; 43: 943-956.

17. Monis PT, et al. Variation in Giardia: towards a taxonomic revision of the genus. Trends in Parasitology 2009; 25: 93-100.

18. Atherton R, et al. Molecular identification of Giardia duodenalis in Ecuador by polymerase chain reactionrestriction fragment length polymorphism. Mémorias do Instituto Oswaldo Cruz 2013; 108: 512-515.

19. Ramírez JD, et al. Molecular diagnosis and genotype analysis of Giardia duodenalis in asymptomatic children from a rural area in central Colombia. Infection Genetics and Evolution 2015; 32: 208-213.

20. Volotão AC, et al. Genotyping of Giardia duodenalis from human and animal samples from Brazil using $\beta$-giardin gene: a phylogenetic analysis. Acta Tropica 2007; 102: 258-262.

21. Kohli A, et al. Giardia duodenalis assemblage, clinical presentation and markers of intestinal inflammation in Brazilian children. Transactions of the Royal Society of Tropical Medicine and Hygiene 2008; 102: 718-725.

22. Durigan M, et al. Genetic diversity of Giardia duodenalis: multilocus genotyping reveals zoonotic potential between clinical and environmental sources in a metropolitan region of Brazil. PLoS ONE 2014; 9: 115-489.
23. Colli CM, et al. Identical Assemblage of Giardia duodenalis in humans, animals and vegetables in an urban area in Southern Brazil indicates a relationship among them. PLoS ONE 2015; 10: 118-165.

24. Souza SL, et al. Molecular identification of Giardia duodenalis isolates from humans, dogs, cats and cattle from the state of São Paulo, Brazil, by sequence analysis of fragments of glutamate dehydrogenase $(g d h)$ coding gene. Veterinary Parasitology 2007; 149: 258-264.

25. Volotão AC, et al. Giardiasis as zoonosis: between proof of principle and paradigm in the Northwestern region of São Paulo State, Brazil. Brazilian Journal of Infectious Diseases 2011; 15: 382-383.

26. David EB, et al. Molecular characterization of intestinal protozoa in two poor communities in the State of São Paulo, Brazil. Parasites and Vectors 2015; 8: 1-12.

27. Brazilian Institute of Geography and Statistics Demographic census (http://www.ibge.gov.br) Accessed 16 August 2010.

28. Garcia LS. Diagnostic Medical Parasitology, 4th edn. Washington: ASM Press, 2001, pp. 1092.

29. Lalle M, et al. Genetic heterogeneity at the giardin locus among human and animal isolates of $G$. duodenalis and identification of potentially zoonotic subgenotypes. International Journal for Parasitology 2005; 35: 207-213.

30. Sulaiman IM, et al. Triosephosphate isomerase gene characterization and potential zoonotic transmission of Giardia duodenalis. Emerging Infectious Diseases 2003; 9: 144-152.

31. Read CM, Monis PT, Thompson RCA. Discrimination of all genotypes of Giardia duodenalis at the glutamate dehydrogenase locus using PCR-RFLP. Infection, Genetics and Evolution 2004; 4: 125-130.

32. Larkin MA, et al. Clustal W and Clustal X version 2.0. Bioinformatics 2007; 23: 2947-2958.

33. Greenland S. Modeling and variable selection in epidemiologic analysis. American Journal of Public Health 1989; 79: 340-349.

34. Cañete R, et al. Intestinal parasites in children from a day care centre in Matanzas City, Cuba. PLoS ONE 2012; 7: 513-594.

35. Prado MS, et al. Risk factors for infection with Giardia duodenalis in pre-school children in the city of Salvador, Brazil. Epidemiology and Infection 2003; 131: 899-906.

36. Mateo M, et al. Detection and molecular characterization of Giardia duodenalis in children attending day care centers in Majadahonda, Madrid, Central Spain. Medicine (Baltimore) 2014; 93: 15-75.

37. Silva RR, et al. Association between nutritional status, environmental and socio-economic factors and Giardia lamblia infections among children aged 6-71 months in Brazil. Transactions of the Royal Society of Tropical Medicine and Hygiene 2009; 103: 512-519.

38. Lalle $\mathbf{M}$, et al. High genetic polymorphism among Giardia duodenalis isolates from Sahrawi children. Transactions of the Royal Society of Tropical Medicine and Hygiene 2009; 103: 834-838.

39. Broglia A, et al. Molecular typing of Giardia duodenalis isolates from German travellers. Parasitology Research 2013; 112: 3449-3456. 
40. Kuk S, et al. Stool sample storage conditions for the preservation of Giardia intestinalis DNA. Mémorias do Instituto Oswaldo Cruz 2012; 107: 965-968.

41. Lebbad M, et al. Dominance of Giardia assemblage B in León, Nicaragua. Acta Tropica 2008; 106: 44-53.

42. Puebla LJ, et al. Correlation of Giardia duodenalis assemblages with clinical and epidemiological data in Cuban children. Infection, Genetics and Evolution 2014; 23: 7-12.

43. Read C, et al. Correlation between genotype of Giardia duodenalis and diarrhoea. International Journal for Parasitology 2002; 32: 229-231.

44. Aydin AF, et al. Classification of Giardia duodenalis parasites in Turkey into groups A and B using restriction fragment length polymorphism. Diagnostic Microbiology and Infectious Disease 2004; 50: 147-151.

45. Almeida A, et al. Genotype analysis of Giardia isolated from asymptomatic children in northern Portugal. Journal of Eukaryotic Microbiology 2006; 53: 177-178.
46. Sahagún J, et al. Correlation between the presence of symptoms and the Giardia duodenalis genotype. European Journal of Clinical Microbiology and Infectious Diseases 2008; 27: 81-83.

47. Haque R, et al. Prospective case-control study of the association between common enteric protozoal parasites and diarrhea in Bangladesh. Clinical Infectious Diseases 2009; 48: 1191-1197.

48. Van Lithh L, et al. A real-time assemblage-specific PCR assay for the detection of Giardia duodenalis assemblages A, B and E in fecal samples. Veterinary Parasitology 2015; 211: 28-34.

49. Wiellinga C, et al. Multi-locus analysis of Giardia duodenalis intra-Assemblage $\mathrm{B}$ substitution patterns in cloned culture isolates suggests sub-Assemblage B analyses will require multi-locus genotyping with conserved and variable genes. International Journal for Parasitology 2011; 41: 495-503. 